\title{
Videoendoscopia em equinos ao alcance todos: os recursos do smartphone
}

\section{Equine video endoscopy available to all: another smartphone tool}

\author{
Gustavo Henrique Zimmermann Winter ${ }^{1^{*}}$, Luis Felipe Pelinson Passos ${ }^{2}$, Andreza Morais de Souza ${ }^{1}$ \\ ${ }^{1}$ Universidade Federal do Rio Grande do Sul (UFRGS), Porto Alegre, RS, Brasil \\ ${ }^{2}$ Centro Universitário Ritter dos Reis (UniRitter), Porto Alegre, RS, Brasil
}

\section{Resumo}

O diagnóstico por imagem é ferramenta importante no dia a dia da clínica de equinos. Os equipamentos, no entanto, requerem um alto capital imobilizado. 0 presente trabalho idealiza e apresenta uma alternativa de baixo custo e alta praticidade para a realização de videoendoscopia em equinos através do uso de mini câmeras acopladas ao smartphone. A partir de um dispositivo Android ${ }^{\circledR}$ (tablet ou smartphone), uma sonda flexível e um boroscópio (câmera USB) é possível montar um videoendoscópio para uso no campo. 0 presente artigo ensina como montar o videoendoscópio, suas vantagens e desvantagens, e apresenta exemplos das imagens visualizadas. Nos exames realizados em equinos foi posível visualizar as vias aéreas, parte do sistema digestivo e parte do útero nas éguas. Entende-se que a videoendoscopia aqui apresentada é um interessante recurso de triagem diagnóstica ao médico veterinário de campo, bem como em uso na educação do acadêmico em medicina veterinária ou cursos de educação continuada.

Palavras-chave: Endoscopia. Sistema respiratório. Vias aéreas. Semiologia. Cavalo.

\begin{abstract}
Diagnostic imaging is an important tool to the equine practitioner. The equipment, however, needs a high investment. The present work suggests a practical and lowcost alternative for performing video endoscopy in horses through the use ofmini-cameras coupled to the smartphone. With Android ${ }^{\circledR}$ devices (tablet or smartphone), a flexible tube and a borescope (USB camera) one can mount a video device for use in the clinics. The present article teaches how to assemble the video endoscope, its advantages and disadvantages, and presents examples of the acquired images. In the exams performed on horses, it was possible to visualize the airways, part of the digestive system, and the uterus in mares. We suggest that the video endoscopy presented here is an interesting diagnostic screening tool for the equine practitioner, as well as in the education of veterinary scholars or continuing education courses.
\end{abstract}

Keywords: Endoscopy. Respiratory system. Airways. Semiology. Horse. 


\section{Introdução}

0 diagnóstico por imagem é ferramenta importante no dia a dia da clínica de equinos. Alguns equipamentos, no entanto, necessitam alto capital imobilizado e por vezes demoram em proporcionar retorno financeiro. Entre estes, destacam-se os equipamentos de radiologia digital, ultrassonografia e videoendoscopia.

Na clínica médica veterinária, a endoscopia, com ou sem vídeo acoplado, é empregada como recurso semiológico na visualização direta, minimamente invasiva, em diversos sistemas do corpo (Slovis, 2004). Em equinos tem destaque o exame das vias aéreas pela rinoscopia, laringoscopia e a traqueobroncoscopia: desde a cavidade nasal até os brônquios (Roy e Lavoie, 2003; Slovis, 2004; Barakzai, 2007). Podem ser utilizadas, ainda, a gastroscopia (estômago), a histeroscopia (útero), a uretroscopia (uretra no garanhão), a cistoscopia (bexiga urinária), a proctoscopia (ânus e reto) e a otoscopia (conduto auditivo externo). No garanhão também podem ser visualizadas por endoscopia as vesículas seminais (Slovis, 2004).

Em cavalos, a endoscopia requer, via de regra, o uso de um endoscópio flexível acoplado a uma fonte de luz, com ou sem conexão ao monitor de vídeo (Slovis, 2004). Um videoendoscópio fornece maior qualidade de imagem do que o fibroendoscópio e permite que vários observadores acompanhem o exame ao mesmo tempo (Roy e Lavoie, 2003). De maneira geral, estes equipamentos custam entre $\mathrm{R} \$$ 35 mil e R\$ 85 mil dependendo da marca, modelo, espessura e comprimento do endoscópio. Um modelo disponível para animais de pequeno porte pode custar a partir de $\mathrm{R} \$ 17$ mil. Para exames de broncoscopia, histeroscopia e cistoscopia, as sondas com $160 \mathrm{~cm}$ de comprimento são apropriadas. No entanto, para a gastroscopia no cavalo adulto é imprescindível a utilização de endoscópios de 240 cm de comprimento.

0 presente trabalho apresenta uma alternativa de baixo custo e alta praticidade para a realização de videoendoscopia em equinos através do uso de mini câmeras acopladas ao smartphone. Idealizouse que através do uso de câmeras para smartphones e tablets com sistema Android $^{\circledR}$ e aplicativos específicos é possível realizar procedimentos de videoendoscopia em odontologia, gastroscopia e vias aéreas superiores e inferiores.

A câmera em questão é uma mini câmera, conhecida como boroscópio, que tem sido utilizada há anos na manutenção mecânica aeronáutica. 0 termo boroscópio vem do inglês borescope e se refere ao método de visualizar (do grego skopein) estruturas internas através de um pequeno furo (do inglês bore); procedimento que é referido como endoscopia na área médica.

\section{Material e métodos}

\section{Equipamentos, partes e requisitos}

Para a montagem deste equipamento de videoendoscopia são necessários alguns itens: um dispositivo Android ${ }^{\circledR}$ como tablet ou smartphone, uma sonda flexível, mas rígida o suficiente para introdução do endoscópio no paciente, e o próprio boroscópio ou câmera USB.

0 dispositivo Android ${ }^{\circledR}$ pode ser de diversas gerações (4.0 ou superior), tanto de smartphones quanto de tablets. Um requisito indispensável é a capacidade OTG da entrada mini USB. OTG é a conhecida função on the go, que permite a troca de dados e não somente energia através da entrada USB; desta maneira, é possível também conectar um pendrive pelo USB e transferir os dados no smartphone/tablet. $\mathrm{O}$ utilizador pode checar se o seu dispositivo possui esta função por aplicativos (app) como o USB-OTG Checker ou similares.

No dispositivo Android é necessária a instalação de um aplicativo básico para formação da imagem captada. Este app fará o reconhecimento da câmara USB do boroscópio. Neste estudo foram utilizados diversos app gratuitos, como MScopes, USB Camera e CameraFi . Cada app possui recursos básicos para, além da captura, salvar imagens e vídeos no tablet ou smartphone. Existe, também, a possibilidade de conexão do boroscópio diretamente ao computador (PC). Neste caso, pode ser necessário o adaptador de mini-USB para USB tamanho padrão nos computadores. Nos exames em que foi utilizado o PC, a imagem foi adquirida pelo programa de câmera do Windows ${ }^{\circledR}$, sem a necessidade de outros softwares. 
A sonda flexível indicada neste estudo é uma sonda nasogástrica comumente utilizada na clínica de equídeos, com diâmetro interno de pelo menos $11 \mathrm{~mm}$. Esta sonda tem a função de uma estrutura externa que promova a rigidez necessária sem causar muito desconforto ou lesões no paciente, assim permitindo a colocação, direcionamento e alcance, manipulação e, consequentemente, visualização das estruturas de interesse.

Para exames como a histeroscopia em éguas, propõe-se o uso de sonda de silicone com balonete, as mesmas utilizadas nas lavagens uterinas e coletas de embrião. Geralmente a sonda com diâmetros maiores que $\mathrm{CH} 32$ já permitem a passagem do boroscópio.

O boroscópio nada mais é do que um acessório com câmera digital para capturar imagens em um fio ou cabo de comprimento variado. Em geral, são encontrados modelos de $50 \mathrm{~cm}$ até $10 \mathrm{~m}$ de comprimento. 0 diâmetro máximo é dado pela lente e quantidade de LEDs embutidos para iluminação, variando de 5 a $8 \mathrm{~mm}$.
Neste estudo foram utilizados boroscópios com seis LEDs, diâmetros de 7 ou 8 mm, câmera HD com resolução variando de 640 x 480 pixels para uso no dispositivo Android ${ }^{\circledR}$ até 720 pixels no PC, e comprimentos de $2 \mathrm{~m}, 3,5 \mathrm{~m}$ e $5 \mathrm{~m}$. Esta mini câmera, ou boroscópio, possui conexão mini USB para conexão ao dispositivo Android ${ }^{\circledR}$. Pode ser adquirida em seus diversos modelos à pronta entrega em vários sites de vendas, por valores entre $\mathrm{R} \$ 29$ e $\mathrm{R} \$ 80$.

\section{Montagem do equipamento}

Para uso efetivo do videoendoscópio aqui proposto é realizada a colocação do boroscópio no interior da sonda (nasogástrica ou uterina) até que a lente alcance a extremidade distal da sonda (Figura 1A). Em seguida, conecta-se a porta mini USB do tablet ou smartphone, momento em que já se acendem os LEDs luminosos. Tão logo seja inicializado o app, já se tem a imagem formada e pode-se proceder o exame (Figura 1B).

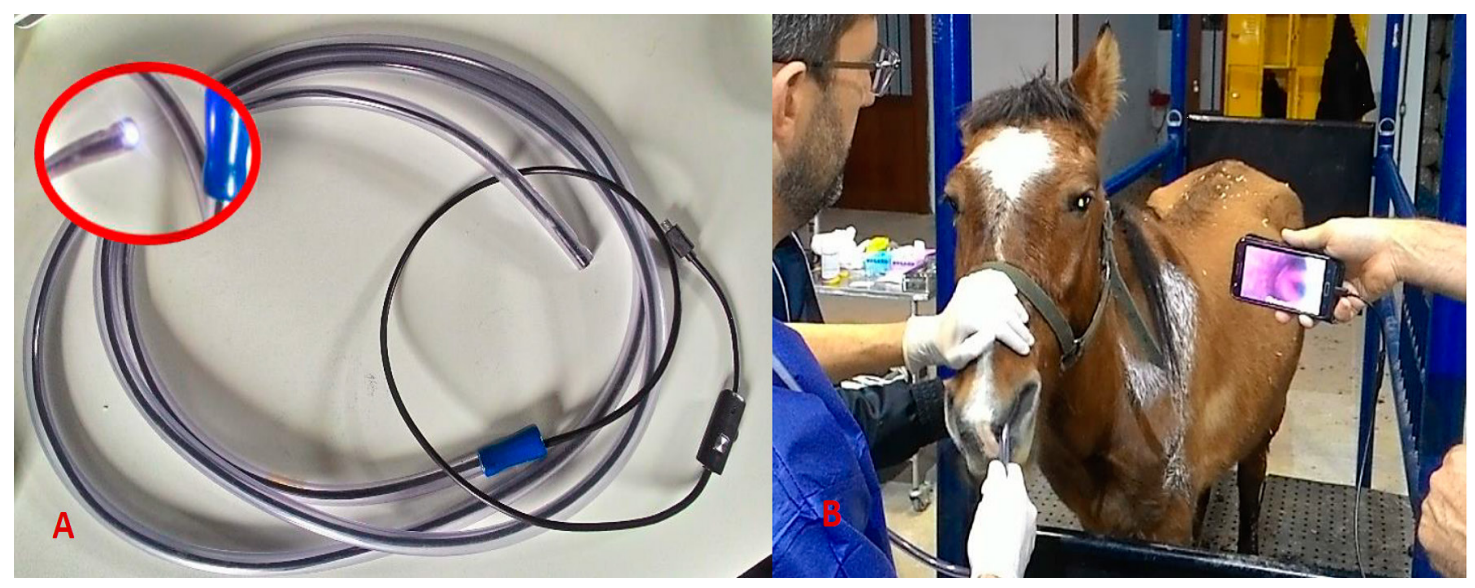

Figura 1 - (A) Boroscópio inserido no interior de uma sonda nasogástrica. No detalhe, a posição da lente e LEDs acesos. (B) Procedimento de videoendoscopia em equino. Na tela do smartphone, pode-se notar a visualização da região da faringe dorsalmente às cartilagens aritenoides.

\section{Exames e procedimentos}

A preparação do paciente requer a contenção específica em troncos ou bretes apropriados aos equinos. Dependendo do temperamento do paciente, é preconizada a utilização de contenção física mais específica, como os uso do "cachimbo" ou prega cutânea, por exemplo. Eventualmente, a contenção farmacológica com uso de fármacos $\alpha 2$-agonistas é necessária. Para tanto, tem sido indicado o uso de xilazina ou detomidina cerca de cinco minutos antes do exame (Barakzai, 2007). A escolha da dose deve 
levar em conta o temperamento e a atitude inicial do paciente em procedimentos que nada diferem de uma endoscopia tradicional.

Na histeroscopia, o videoendoscópio deve ser posicionado nolúmen uterino (Slovis, 2004). Oútero, por sua vez, precisa ser distendido previamente com gás ou líquido. Recomendamos o uso de solução fisiológica $(\mathrm{NaCl}$ 0,9\%) para a distensão uterina em volumes de 500 a $1000 \mathrm{ml}$ (ou mais se necessário). Utiliza-se uma sonda uterina, tanto para proceder a infusão de líquido como para a colocação da mini câmera. Os cuidados de antissepsia do paciente e do instrumental utilizado, bem como a contenção em tronco ou brete para equinos, são imperativos. A passagem do videoendoscópio se dá por procedimento similar às lavagens uterinas.

0 uso em procedimentos de odontologia foi preconizado pela equipe do estudo em cursos da área. Uma pequena modificação da montagem do videoendoscópio é a possibilidade de fixação da mini câmera diretamente em uma caneta odontológica, ou espelho, para posicionamento no interior da boca do paciente. Optou-se, neste estudo, por conectar o boroscópio a um computador, e este foi conectado via HDMI a um monitor de 42 polegadas. Todos os demais procedimentos preconizados nos procedimentos em odontologia em equinos foram mantidos.

0 material utilizado na montagem da sonda e o boroscópio são resistentes à água, de modo que sempre podem ser higienizados, e deve ser procedida a antissepsia prévia aos exames de broncoscopia e histeroscopia. Recomenda-se a utilização de soluções à base de clorexidina $2 \%$, seguida de água estéril ou solução fisiológica para a antissepsia.

\section{Resultados}

\section{Exames realizados}

Nos exames realizados com esta montagem de videoendoscopia por smartphone em equinos não foi necessária a sedação dos pacientes. Eventualmente, para a introdução da sonda nasogástrica, fez-se necessária a contenção física momentânea com prega cutânea sobre a região do pescoço e paleta. A luz proveniente dos LEDs da sonda não alterou o comportamento dos pacientes, não tendo sido notados movimentos bruscos típicos de susto ou medo.

Uma vez inserida a sonda, pode-se optar por uma endoscopia de vias aéreas superiores, laringe, traqueia e brônquios, ou promover a deglutição e passagem esofágica até o estômago. Na endoscopia de vias aéreas foi notável a qualidade e clareza das imagens obtidas nas diversas regiões anatômicas (Figura 2). A identificação do septo e conchas nasais, bem como da laringe e suas cartilagens, deuse sem complicações, com raros embaçamentos momentâneos da lente. 0 nível de iluminação dado pelo LED é suficiente para a adequada formação da imagem.

Pela passagem via esofágica e utilização de sonda nasogástrica longa foi possível chegar ao estômago. A visualização da mucosa estomacal permitiu a identificação de gastrite e úlceras. Rapidamente, porém, a lente foi encoberta por conteúdo da ingesta e suco gástrico, dificultando a avaliação mais precisa do estômago; uma evidente desvantagem de não ser possível a limpeza remota da lente neste equipamento. Durante a passagem do endoscópio pelo esôfago, a clareza das imagens foi obtida sem que acontecessem obstruções na lente (Figura 2G e H).

Nos procedimentos de histeroscopia, foi possível a visualização do fundo de saco vaginal e cérvice pela simples condução do boroscópio pela mão enluvada via vaginal. Para a passagem ao útero, foi imperativo o auxílio da sonda uterina e do prévio preenchimento e distensão do lúmen uterino com solução salina. Neste procedimento, pode-se optar por utilizar a sonda uterina para infusão da solução e posterior passagem do boroscópio pela sonda que permanece na cérvice, ou utilizar duas sondas uterinas, uma delas previamente montada como o boroscópio para substituir a sonda utilizada anteriormente na distensão do lúmen uterino com solução fisiológica. Uma vez dentro do útero, o videoendoscópio revelou imagens de boa qualidade do endométrio do corpo uterino, bifurcação e entrada dos cornos uterinos. Foi preciso, apenas, a inserção no sentido cranial da sonda e leves movimentos de rotação para tentar direcionar a lente mais à esquerda ou à direita. 


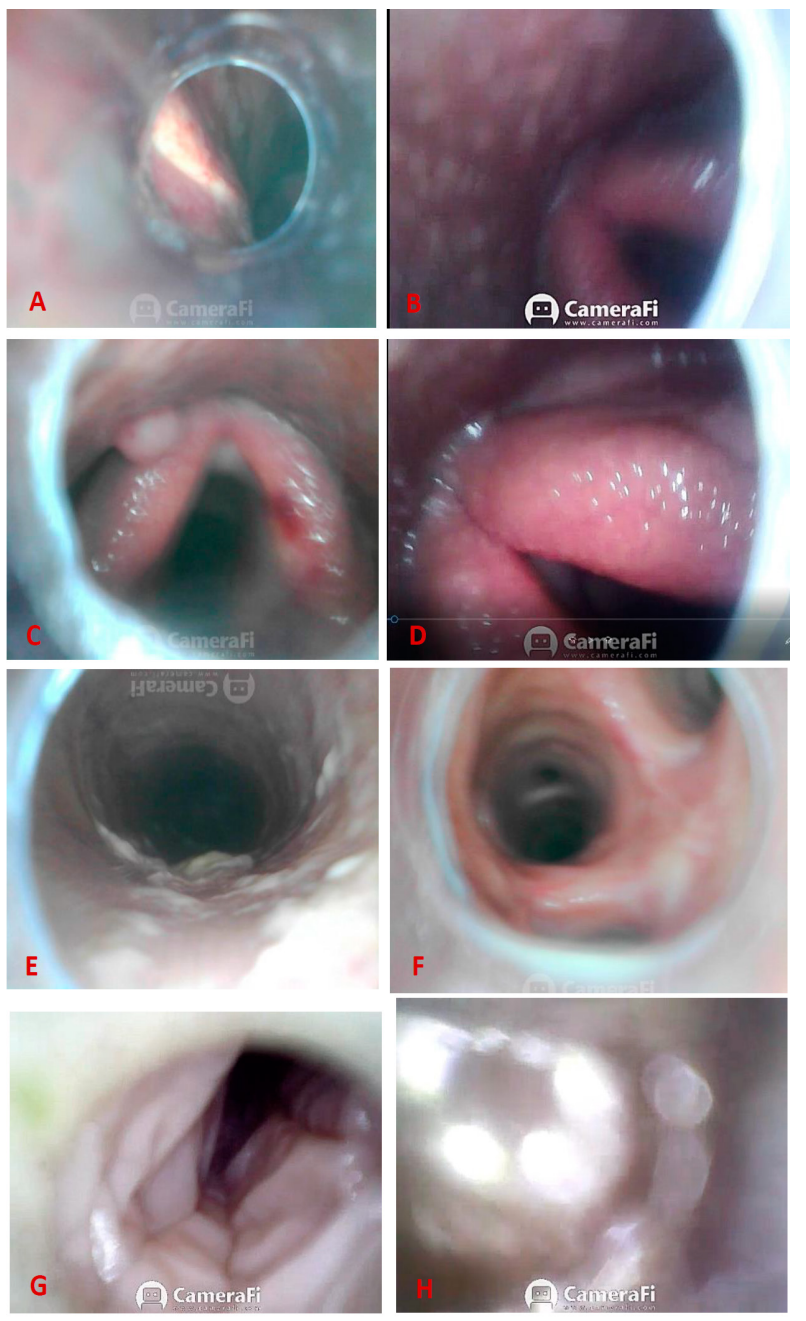

Figura 2 - Videoendoscopia das vias aéreas e esôfago no equino, permitindo visualização de (A) membrana faringeal da bolsa gutural direita, (B) hiperplasia linfoide faríngea, $(C, D)$ cartilagens aritenoides, $(E)$ traqueia, $(F)$ carina e brônquios, $(G)$ mucosa esofágica e $(H)$ obstrução da lente pelo conteúdo estomacal.

Para a avaliação odontológica é imperativo o uso de um abre-bocas de cremalheira ou similar. Como parte do presente estudo, a utilização da videoendoscopia da cavidade oral foi demonstrada em um curso prático de odontologia equina. Neste caso, o equipamento foi acoplado ao PC que, por sua vez, estava ligado a um monitor de 42 polegadas (televisor convencional) pela conexão HDMI. Durante os procedimentos de avaliação e terapêutica nos dentes dos pacientes, foi muito clara a grande visibilidade dada pelo endoscópio no monitor (Figura 3).

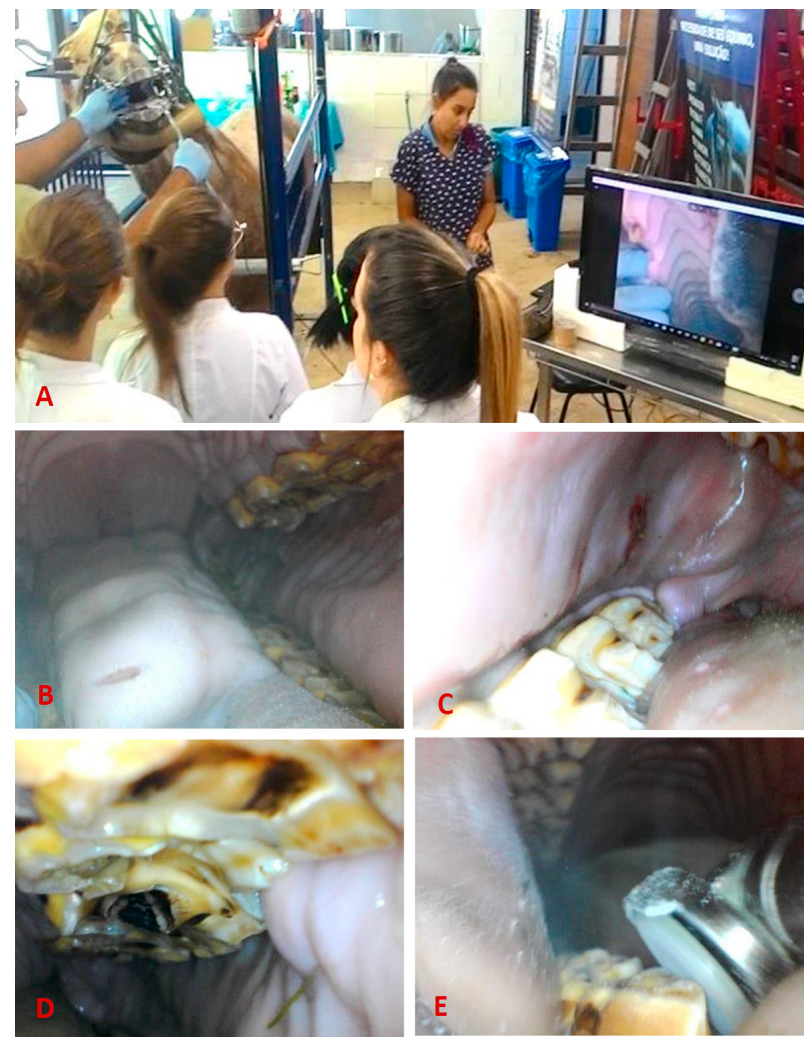

Figura 3 - Procedimento de videoendoscopia utilizado como método auxiliar de avaliação bucal odontológica em equino. (A) Procedimento no paciente contido e sedado; equipamento acoplado ao PC e monitor. (B, C e D) Inspeção e avaliação bucal odontológica. (E) Momento da correção de gancho dentário.

\section{Vantagens da videoendoscopia pelo smartphone}

Entre as principais vantagens deste sistema de videoendoscopia, destacam-se: (1) valor reduzido quando comparado ao endoscópio médico; (2) resistência à poeira e à água (IP67 ou IP68), podendo-se realizar higienização e antissepsia com soluções como clorexidina; (3) boa qualidade de imagem (HD) com iluminação LED embutida; (4) possibilidade de salvar fotos e vídeos; (5) portátil e de diâmetro reduzido para introdução na sonda nasogástrica comercial; (6) modelos com diversos comprimentos $(1 \mathrm{~m}, 1,5 \mathrm{~m}, 2 \mathrm{~m}, 5 \mathrm{~m}$ e até $10 \mathrm{~m})$, o que permite endoscopia pulmonar, gastroscopia e histeroscopia com segurança, e ergonomia dos envolvidos; (7) facilidade de utilização pelo operador, no smartphone, com disponibilidade de 
aplicativo gratuito; (8) agrega valor ao serviço do médico veterinário de equinos ao mesmo tempo que impacta como moderna ferramenta de diagnóstico perante o cliente; (9) é uma ferramenta de auxílio diagnóstico ou educacional.

\section{Desvantagens}

Como principais desvantagens deste sistema, citam-se: (1) não permite controle/orientação da lente por não possuir as rodas de comando para o mecanismo de deflexão angular da extremidade distal da sonda; (2) não permite limpeza remota da lente por não possuir válvulas de ar ou água; (3) não possui canal de trabalho para sondas e pinças, como os endoscópios médicos possuem. Alguns modelos possuem ainda a função de aspiração (sucção) pelo canal de trabalho, que não é o caso do boroscópio aqui apresentado. Ainda, por ser bastante fino e flexível, o boroscópio requer o uso de sonda nasogástrica que seja igualmente acessível e de baixo custo.

\section{Discussão e conclusão}

Este endoscópio se mostrou eficaz como auxílio em atividades de ensino, cursos técnicos e como ferramenta de diagnóstico rápido, eficiente, portátil e de baixo custo, apresentando uma importante aplicabilidade como triagem diagnóstica de campo. É importante ressaltar que este videoendoscópio não contempla todas, e nem visa substituir, as funcionalidades de um equipamento de endoscopia flexível convencional, como o importante direcionamento da lente e canais de trabalho. Demonstrou-se, porém, um válido recurso semiológico numa triagem diagnóstica, como proposto pela mesma equipe em divulgação prévia (Winter et al., 2017), além de contar com a funcionalidade de gravar fotos e vídeos para laudos e envio em consultas a outros profissionais.

A simples possibilidade de expansão dos meios diagnósticos dos exames de imagem para níveis até então somente disponíveis a poucos clínicos especializados ou centros de referência é um evidente ganho da técnica aqui apresentada.

As enfermidades do sistema respiratório contemplam uma miríade de diagnósticos diferenciais, cada qual com sua conduta terapêutica específica (Higgins e Snyder, 2006). Uma correta avaliação do quadro clínico do paciente e a realização de exames complementares ganha, com este videoendoscópio acessível pelo smartphone, novo alcance como triagem da gravidade do caso e indicação de necessidade de outras investigações; ou mesmo, temse potencialmente a possibilidade de diagnósticos clínicos definitivos pela confirmação ou exclusão de enfermidades após a videoendoscopia. Do mesmo modo, os exames obtidos pela histeroscopia e esofagoscopia se mostraram eficientes para a obtenção das imagens se comparados à literatura específica (Slovis, 2004).

Este equipamento se mostrou uma boa e adequada ferramenta de diagnóstico por imagem para exame a campo objetivando triagem e auxílio diagnóstico. Apresentou boa qualidade de imagem e acurácia semiológica, principalmente nas vias aéreas. Isto confere um potencial de acelerar o processo diagnóstico e a correta conduta clínica veterinária oferecida ao paciente. Além de seu uso na educação do acadêmico em medicina veterinária ou em cursos de educação continuada, esta ferramenta é uma bela alternativa para uso em países subdesenvolvidos, onde os recursos em geral são mais escassos.

\section{Referências}

Barakzai S. Handbook of equine respiratory endoscopy. Philadelphia: Saunders; 2007. 144 p.

Higgins AJ, Snyder JR. The equine manual. 2 ed. Philadelphia: Saunders; 2006.1460 p.

Roy MF, Lavoie JP. Tools for the diagnosis of equine respiratory disorders. Vet Clin North Am Equine Pract. 2003;19(1):1-17.

Slovis NM. Atlas of equine endoscopy. St Louis: Mosby; 2004. 254 p.

Winter GHZ, Passos LFP, Souza AM. Videoendoscopia ao alcance de todos: os recursos do smartphone. XVIII Conferência Anual da ABRAVEQ; 2-4 jun 2017; Águas de Lindóia, SP. Rev Acad Cienc Anim. 2017;15(Supl.1):S121-2. 